\title{
A meta-analysis on genetic variability of RT/ HBsAg overlapping region of hepatitis B virus (HBV) isolates of Bangladesh
}

\author{
Md. Golzar Hossain ${ }^{1,2^{*}}$ (i) and Keiji Ueda ${ }^{1 *}$
}

\begin{abstract}
Background and aim: Hepatitis B caused by HBV is a serious public health hazard prevalent worldwide including Bangladesh. Few scattered molecular studies of HBV have been reported in Bangladesh. This study aimed to analyze the genetic variability of RT/HBsAg overlapping region of HBV isolates of Bangladesh and determination of correlation among the genotype/serotype and HBsAg escape and/or drug-resistant mutations.

Methods: A total of 97 complete HBsAg sequences of Bangladeshi HBV isolates from 2005 to 2017 from NCBI GenBank were extracted and analyzed using several HBV bioinformatics tools such as Geno2pheno-HBV, HBV Serotyper, HIV-Grade:HBV-Tool, and CLC sequence viewer.

Results: The prevalence of genotypes A, C, and D are 18, 46 and 35\% which correspond to serotype adw, adr, and ayw, respectively. The prevalence of HBsAg escape mutations is $51 \%$ and most of which (62\%) are found in the genotype $D$ followed by $32 \%$ in genotype $C$ and $6 \%$ in genotype A. Interestingly most (24/36) of the sequences of HBsAg escape mutations contained $128 \mathrm{~V}$ mutant which all belongs to only serotype ayw3 (Genotype D). Prevalence of drug-resistant mutations is $\sim 11 \%$, most of which are from genotype $C(63.64 \%)$ and $D(36.36 \%)$. Lamivudine resistant mutations were found in 11\% of sequences followed by Telbivudine 10\% and Adefovir 3\% where Tenofovir showed susceptibility to all 97 sequences. Moreover, 7 among of 97 sequences showed both $\mathrm{HBsAg}$ and drugs resistant mutations and none of them are found due to the same nucleotide substitutions.

Conclusion: There is a strong correlation among the genotype/serotype and HBsAg escape and/or drug-resistant mutations. This meta-analytical review will be helpful for genotype-serotype prediction by PCR-based diagnosis and development of vaccine and/or diagnostic kits, and the treatment against HBV infection in the future.
\end{abstract}

Keywords: Hepatitis B virus (HBV), Serotype, Genotype, HBsAg escape mutations, Drug-resistant mutations, Bangladesh

\section{Introduction}

Hepatitis B is caused by one of the smallest enveloped DNA viruses known as HBV under the family of hepadnaviridae. HBV can transmit vertically (mother to baby) and by horizontally such as sexual contact, sharing needles, syringes, razors, and blood transfusion, etc., $[1,2]$. HBV causes both acute and chronic infections. Liver failure may occur in some cases of acute HBV infection which leads to sudden death. About $15-25 \%$ people of

\footnotetext{
* Correspondence: mghossain@bau.edu.bd; mghossain@virus.med.osakau.ac.jp; kueda@virus.med.osaka-u.ac.jp

${ }^{1}$ Division of Virology, Department of Microbiology and Immunology, Graduate School of Medicine, Osaka University, Osaka, Japan Full list of author information is available at the end of the article
}

the chronically HBV infected patients may develop liver cirrhosis followed by hepatocellular carcinoma (HCC). However, liver cirrhosis and HCC may become more worsen due to coinfection with the hepatitis delta virus (HDV) and HBV because of using HBV envelope proteins by HDV for replication [3].

HBV genome is a partially double-stranded circular DNA of $3.2 \mathrm{~kb}$ in size. Four open reading frames (ORFs): $\mathrm{P}, \mathrm{C}, \mathrm{S}$, and $\mathrm{X}$ are encoded by the viral genome. Small HBsAg envelope protein produced by the common Cterminal domain of ORF S, composed of 226 amino acids entirely overlapped with reverse transcriptase (RT) domain of viral polymerase ( $\mathrm{Pol})$ protein produced by 
ORF P [4]. There are four major subtypes (Serotypes) of HBsAg; $a d w, a y w, a d r$, and ayr defined by a common "a" determinant and two mutually exclusive determinants pairs, $\mathrm{d} / \mathrm{y}$ and $\mathrm{w} / \mathrm{r}$ at the positions 122 and 160, respectively $[5,6]$. Clinical diagnosis of $\mathrm{HBV}$ infection is primarily based on the detection of HBsAg whereas recombinant $\mathrm{HBsAg}$ is used as a vaccine $[7,8]$. Because of lack of Pol proofreading activity, mutation frequently occurs of HBV genome including the ORF for $\mathrm{HBsAg}$ protein that leads to $\mathrm{HBsAg}$ detection failure (diagnostic escape) by conventional routine diagnostic HBsAg ELISA and vaccination failure (Vaccine escape) [9-14]. On the other hand, many drug resistant mutations in the RT domain of Pol has been identified. The HBsAg coding sequence completely overlapped on the RT domain of HBV polymerase laying from amino acid position rt10 to rt234 [9, 11, 15, 16]. The RT/HBsAg overlapping region contained majority of the nucleotide conservation and the YMDD (amino acids rt203 to rt206) motif on the RT domain is very important for its function also laid within $\mathrm{HBsAg}$ coding region ( $\mathrm{HBs} \mathrm{Ag}$ nt582 to nt593; Accession No.: JQ514509.1) [15, 17]. Moreover, most of the mutants resistant to anti-HBV drugs are within this $\mathrm{RT} / \mathrm{HBsAg}$ overlapped sequence [18]. Higher rate of mutations in the RT/HBsAg overlapping region correlates with the lower level of serum HBD DNA and HBsAg which attract the researchers to focus on this topic [19].

Hepatitis B is prevalent worldwide including the WHO South-Asian region with a tentatively $2 \%$ of infection among the general people. It is also prevalent in Bangladesh ranging from 0.8 to $6.2 \%$ [20-22]. Few molecular analysis of HBV genetic analyses in Bangladesh have been performed with a narrow time period with limited regions [22-24]. These studies were limited to a specific region and or institute and some of them has no drug-resistant analysis or serotype (subtype) determination or HBsAg escape mutations analysis. Moreover, there is no report so far showing the relationship among genotype, serotype (subtype), HBsAg escape, and/or drug-resistant mutations. Therefore, the aim of the metaanalytical review is to determine the genetic variability of $\mathrm{RT} / \mathrm{HBsAg}$ overlapping region of $\mathrm{HBV}$ isolates of Bangladesh and assessment of correlation among the genotype/serotype and HBsAg escape and/or drug-resistant mutations along with their prevalence determination.

\section{Methods}

\section{Collection and processing of HBV sequences from NCBI database}

A total of 97 complete coding sequences of HBsAg were collected from the NCBI GenBank by searching as HBV, Bangladesh under the nucleotide database (Additional file 1). The search results were as "Hepatitis B virus" [Organism] OR HBV [All Fields] AND Bangladesh [All
Fields]. However, we also searched with other keywords but no additional sequences were found. We have taken only the complete HBsAg cds excluding the partial cds. Partial cds would not explain the exact number of mutations in the $\mathrm{HBsAg}$ region overlapped with RT. These HBsAg sequences were from 2005 to 2017 as dated either by sample collection or submission to the GenBank. The nucleotide sequences copied from NCBI were processed in the CLC Sequence viewer (http://www.clcbio.com) for further use.

\section{Determination of serotype (subtype) and genotype}

The genotypes of all these HBsAg sequences were redetermined using the online bioinformatics tool Geno2pheno-HBV (https://hbv.geno2pheno.org). Serotype of some HBsAg sequences submitted from Bangladesh into NCBI GenBank was not mentioned previously and we determined the serotype of those sequences by online HBV Serotyper (http://hvdr.bioinf.wits.ac.za/serotyper/) [25].

\section{Analysis of HBsAg escape and drug-resistant mutation}

The HBsAg escape and drug-resistant mutational analyses were performed using Geno2pheno-HBV (https://hbv. geno2pheno.org) and HIV-Grade:HBV-Tool (https:// www.hiv-grade.de/cms/grade/explanations/hbv-tool/) [26, 27]. The Geno2pheno-HBV provides the HBsAg escape mutation with the mode of action such as vaccine, detection, and therapy escape whereas HIV-Grade:HBV-Tool concludes only as typical mutations for HBsAg escape detected.

\section{Statistical analysis}

Statistical analysis was performed using JavaScript-STAR version BETA-E. To determine the $p$-value among different parameters, Chi-square test was performed and the significance level was considered as 0.05 .

\section{Results}

Prevalence of genotypes and serotypes (subtypes)

The HBV genotype and serotype prevalence among the 97 complete HBsAg coding sequences from the NCBI GenBank during 2005-2017 deposited as Bangladeshi $\mathrm{HBV}$ isolates were determined. Results demonstrated that only three genotypes, A, C, and D are circulating in Bangladesh with 7 subgenotypes A1, A2, C1, C2, D1, D2, and D3 (Fig. 1a). The predominant genotype is C (46.39\%) followed by D (35.05\%) and A (18.56\%). Serotyping analysis showed that the most common subtype is $a d r(44.33 \%)$. The prevalence of serotype ayw (ayw1, ayw2, and $a y w 3$,) and $a d w(a d w 2)$ is 37.11 and $18.56 \%$, respectively. However, within these 97 sequences, there is no report of serotype ayr (Fig. 1b). 

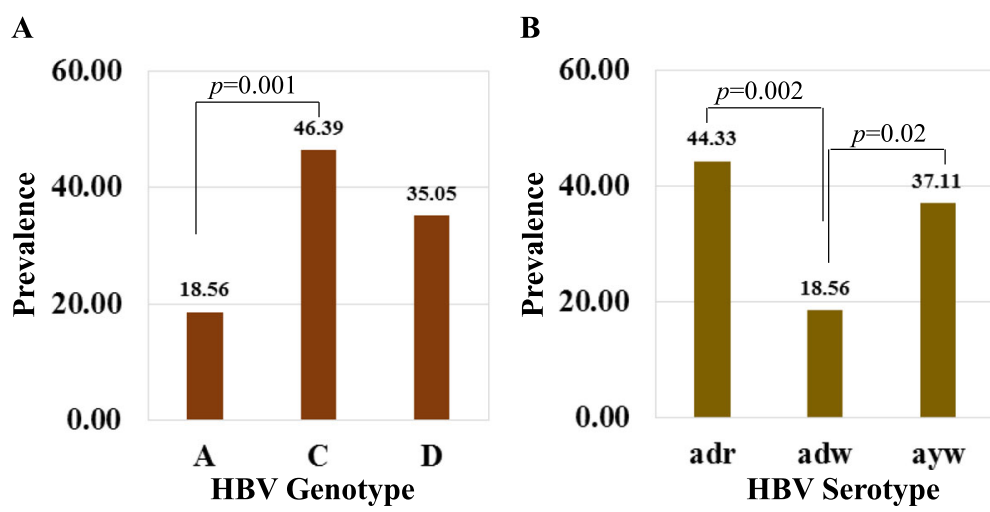

Fig. 1 Prevalence of HBV genotype and serotype. Ninety-seven complete small HBsAg coding sequences of Bangladeshi HBV isolates of year, 2005-2017 were collected from NCBI GenBank. The HBV genotype and serotype were re-determined by using the bioinformatics tool followed by an analysis of their overall prevalence of genotype (a) and serotype (b) circulating in Bangladesh. Chi-square test was performed to determine the statistical significance level and only the $p$-value greater than 0.05 was mentioned

\section{Relationship among the genotypes and subtypes}

We wanted to know whether there is any relationship among the HBV genotypes and serotypes (subtypes) of HBV isolates of Bangladesh during the long times courses from 2005 to 2017. Among the 45 of genotype C sequences, $43(95.56 \%)$ are under subtype $a d r$ and only 2 (4.44\%) are of $a d w$. The 17 (94.44\%) out of total 18 genotype $\mathrm{A}$ is under the serotype $a d w$ and only 1 $(5.56 \%)$ is under $a y w$. In case of genotype D, all 34 (100\%) are under the serotype ayw (Fig. 2). These results strongly suggested that there is a significant relationship between HBV genotypes and serotypes (subtypes) of Bangladeshi isolates such as serotypes $a d r, a d w$, and $a y w$ belongs to genotypes $\mathrm{C}, \mathrm{A}$, and $\mathrm{D}$, respectively.

\section{Analysis and prevalence of $\mathrm{HBsAg}$ escape mutations}

The prevalence of HBsAg escape mutation among the 97 sequences with Geno2pheno-HBV and HIV-Grade: HBV-Tool is 37.11 and $51.55 \%$, respectively (Fig. 3a).

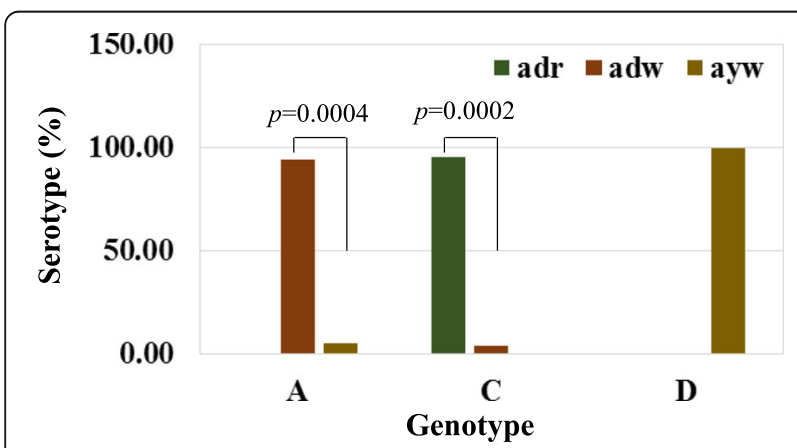

Fig. 2 Distribution of HBV genotype among the serotype. Relationship of HBV genotype and serotype of Bangladeshi HBV isolates from 2005 to 2017 was determined. Chi-square test was performed to determine the statistical significance level and only the $p$-value greater than 0.05 was mentioned
This variation is because Geno2pheno-HBV could not be able to detect T123A, I126T, and P127T as HBsAg escape. However, the most common vaccine escape HBsAg mutant is $128 \mathrm{~V}$. Mutants $120 \mathrm{~T}, 126 \mathrm{~N}$, and $145 \mathrm{R}$ are responsible for the vaccine, therapy (IG), and detection failure. The mutant $100 \mathrm{C}$ and $122 \mathrm{~K}$ are responsible for detection failure. The mutant 126I and143L are responsible for vaccine/therapy (IG) and vaccine/detection, respectively (Fig. 3c).

\section{Analysis of antiviral drug-resistant mutations in RT/ HBsAg overlapping region}

Many drug resistant mutations are found within the RT/ HBsAg overlapping regions [18]. Therefore, we analyzed the anti-HBV drugs resistance of all $97 \mathrm{RT}$ overlapped HBsAg coding sequence isolated from Bangladesh from 2005 to 2017 based on the Geno2pheno-HBV and HIV-Grade:HBVTool. However, results of antiviral drug-resistant mutation in this region from both tools (Geno2pheno-HBV and HIVGrade:HBV-Tool) are similar with negligible difference, and only the results of Geno2pheno-HBV were described here. The overall prevalence of drug-resistant HBV is $11.34 \%$ (11/ 97) where nine (9) sequences showed resistance to at least three anti-HBV drug among lamivudine, adefovir, entecavir, and telbivudine (Fig. 3b). One sequence showed resistant to four drugs lamivudine, adefovir, entecavir, and telbivudine and one showed resistance against only lamivudine. Tenofovir is found to be susceptible for all 97 sequences. However specific drug resistance prevalence is $11.34 \%$ (11/97), 10.30\% (10/97), 8.24\% (8/97), and 3.09\% (3/97) against lamivudine, telbivudine, entecavir, and adefovir, respectively (Fig. 3b).

\section{Relationship of HBsAg escape and drug-resistant mutations with genotype and serotype}

Among the 36 (37.11\%) sequences showing HBsAg escape mutations by Geno2pheno-HBV, the highest 
A

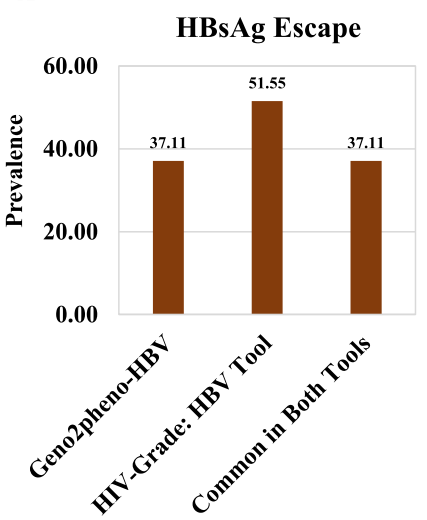

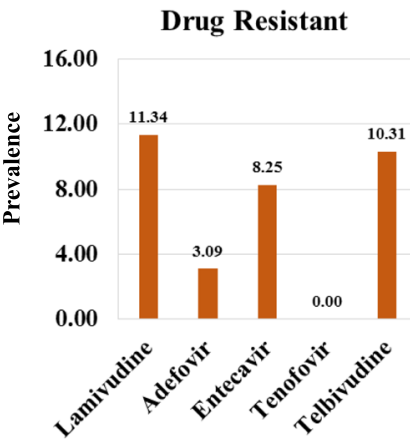

C

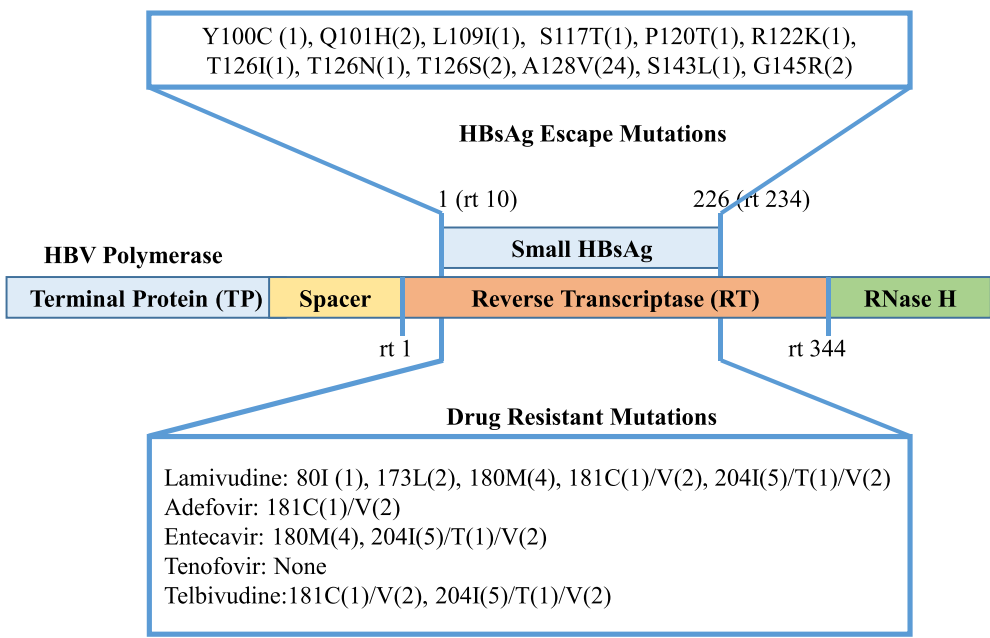

Fig. 3 Prevalence of HBsAg escape and drug-resistant mutations in RT/HBsAg overlapping region. Mutations related to HBsAg escape and drug resistant of RT/HBsAg overlapping region were determined by using Geno2pheno-HBV and HIV Grade: HBV-Tool. a Overall prevalence of HBsAg escape (a) and drug-resistant mutations (b) circulating in Bangladesh from 2005 to 2017 determined by two different bioinformatics tools. c Specific mutations with their frequency related to HBsAg escape and drug-resistant in the RT/HBsAg overlapping region. The numbers within the bracket showed the frequency of that mutation

A

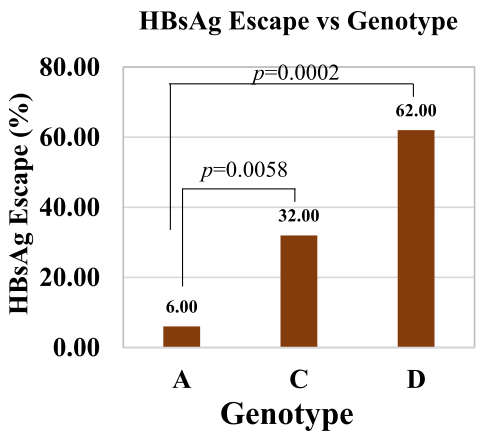

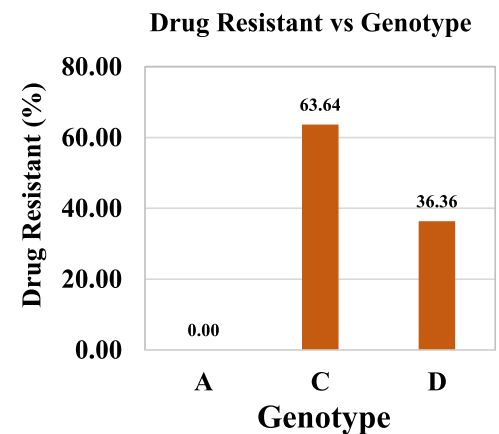

Fig. 4 Distribution (Relationship) of HBsAg escape and drug-resistant mutations among the genotypes. Relationship of HBsAg escape and drugresistant HBV mutations circulating in Bangladesh from 2005 to 2017 was determined (a and b). Chi-square test was performed to determine the statistical significance level and only the $p$-value greater than 0.05 was mentioned 
prevalence of HBsAg mutations was found in genotype D $(69.44 \%, 25 / 36)$ followed by genotype C $(22.22 \%, 8 /$ $36)$ and genotype A $(8.33 \%, 3 / 36)$. Interestingly most (24/36) of the sequences contained $128 \mathrm{~V}$ mutant which all belongs to only serotype ayw3 (Genotype D) though HBsAg amino acid position 128 is not considered for serotype determination (Fig. 4a) [25]. Similar results were found in case of HIV-Grade:HBV-Tool analysis, as HBsAg escape mutations in genotype D $(62 \%, 31 / 50)$ followed by genotype C $(32 \%, 16 / 50)$ and genotype A $(6 \%, 3 / 50)$. The statistical analysis showed that drugresistant mutations are the highest in genotype $\mathrm{C}$ (63.64\%, 7/11) followed by genotype D $(36.36 \%, 4 / 11)$ with absence in genotype A (Fig. 4b). In conclusion, HBsAg escape and drug-resistant mutations are predominant in genotype D (serotype ayw) and genotype C (serotype $a d r$ ), respectively, in Bangladesh. These HBsAg escape or drug-resistant mutations relationship with genotype should correspond with HBV subtypes, as there was a strong correlation of genotypes with the serotype (Subtypes) (Fig. 2).

\section{Relationship between HBsAg escape and drug resistance}

Though 36 or 50 sequences among 97 contained HBsAg escape mutations, we found only 11 or 10 sequences showing drug-resistant mutations by Geno2pheno-HBV and HIV Grade:HBV-Tool, respectively. Only two (2) sequences have HBsAg escape mutations $(128 \mathrm{~V})$ among eleven (11) shown to be resistant against anti-HBV drugs (204I/T and $80 \mathrm{I} /$ ) by Geno2pheno-HBV. On the other hand, six (6) sequences are common having both HBsAg escape and drug-resistant mutations analyzed by HIV Grade:HBV-Tool. One sequence (JQ514498.1) was common in both tools. Therefore, in extend, we analyzed whether the HBsAg escape and drug resistant mutations in these 7 sequences are found from the same nucleotide substitutions. Results demonstrated that none of these mutations are found due to same nucleotide substitutions. However, HBsAg escape and drugs resistant mutations are T123A, I126N/T, P127T, A128V and L80I, $\mathrm{L} 180 \mathrm{M}, \mathrm{A} 181 \mathrm{~V}, \mathrm{M} 204 \mathrm{I} / \mathrm{T} / \mathrm{V}$, respectively, found in these common sequences (Fig. 3c and Fig. 5).

\section{Discussion}

Hepatitis B caused by HBV is an important occupational health hazard. According to WHO, 887 thousand people died in 2015 by liver cirrhosis and HCC induced by HBV. HBV associated HCC has been also reported in Bangladesh and patients infected with genotype $C$ hold the greater risk of HCC development [28, 29]. The HBV genotypic prevalence in Bangladesh was performed scatteredly within a limited time slot [22-24]. In this current meta-analytical review analysis of complete $\mathrm{S}$ gene sequence coding, RT/HBsAg overlapping region based genotyping from NCBI GenBank from 2005 to 2017 showed that genotype $C$ that is predominant $(46.39 \%)$ in Bangladesh correlates with previous studies [22, 23]. Genotype-serotype analysis of Bangladeshi HBV sequences showed a strong correlation such as 95.56, 94.44 , and $100 \%$ of genotypes C, A, and D belong to subtypes $a d r, a d w$, and $a y w$, respectively [30]. The nucleotides diversity determines the HBV genotype and genotype-specific primers designing followed by $\mathrm{PCR}$ can differentiate specific genotypes [23, 31, 32]. Whereas the HBV serotype (subtype) is based on the amino acid positions 122 and 160 of HBsAg [33, 34]. Therefore, this strong correlation between HBV genotype-serotype will be very helpful to predict the subtype by performing PCR only as well as redesigning of diagnostic kit and vaccine strain. Regular analysis and detection of currently circulating $\mathrm{HBsAg}$ escape mutations is very important for diagnostic failure (diagnostic escape), vaccination escape, and inefficient immunoglobulin (HBIG) therapy [35-38]. Almost all of the diagnostic and/or vaccine escape mutations are found in the MHR because of containing highly conformational B-cells epitope cluster which is the major earmark of neutralizing antibodies to HBsAg [10, 39]. Recently developed tools Geno2phenoHBV and HIV-Grade:HBV-Tool are very authentic for the analysis of HBsAg escape and drug-resistant mutations of HBV genome sequence [26, 27]. In our analysis, using these two HBV tools, the prevalence of HBsAg escape mutations in Bangladesh among the 97 sequences from 2005 to 2017 is $37.11 \%$ or $51.55 \%$. Almost all of the HBsAg escape mutations are secondary mutations associated with vaccine or diagnostic escape. Patients harboring HBV infection showing false-negative diagnosis are more harmful and lead to the development of chronic carrier followed by HCC which demands the establishment of new diagnostic kits [40]. Around $2-5 \%$ of the total population in Bangladesh are suffering with chronic HBV infection which require extensive treatment cost [41]. The commonly anti HBV drugs used in Bangladesh are Lamivudine, Adefovir. Entecavir, Tenofovir, and Telbivudine [41]. In our analysis, all of these drugs are resistant at different levels except Tenofovir. Though 8 sequences (8 out of 11 resistant sequences) showed partially resistance against entacavir, only 3 sequences resistant to Adefovir. Lamivudine (11/11) and Telbivudine (10/11) were found to be resistant frequently. As no sequence contained Tenofovir resistant mutations, the treatment strategy needs to replace with Tenofovir instead of others in Bangladesh.

Our prime goal of this review analysis to find out whether there is any statistical relationship between the HBsAg escape and drug-resistant HBV mutations that belong to specific genotype or serotype. Though predominant genotype in Bangladesh is $\mathrm{C}$, higher rate of 
A

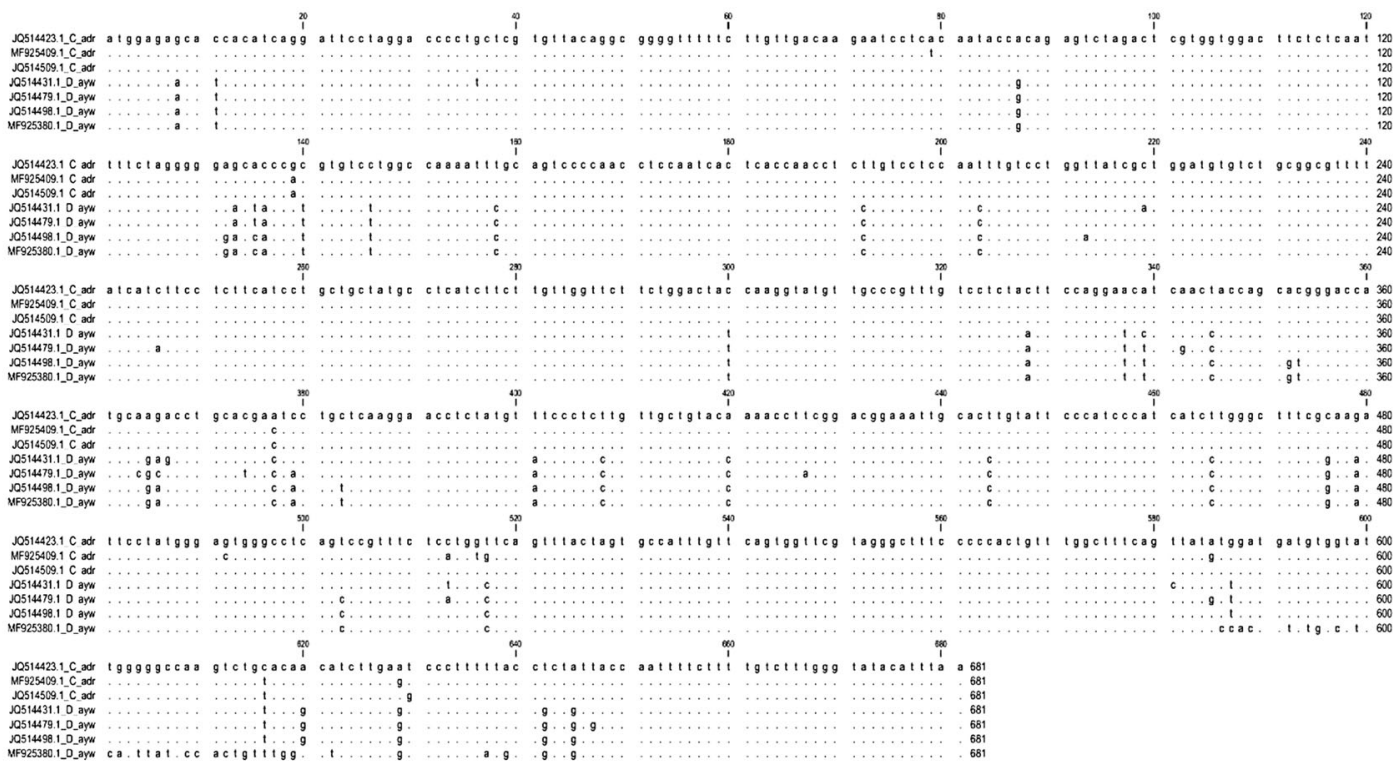

B

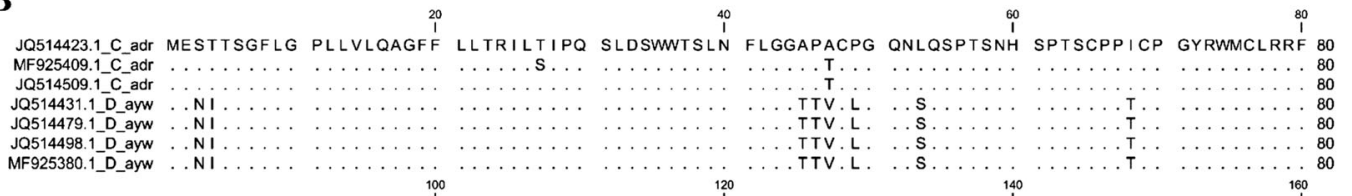

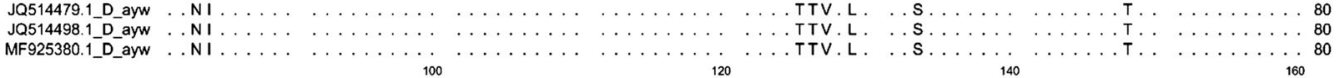
J0514423.1_C_adr IIFLFILLLC LIFLLVLLDY' QGMLPVCPLL PGTSTTSTGP CKTCTNPAQG TSMFPSCCCT KPSDGNCTCI PIPSSWAFAR 160

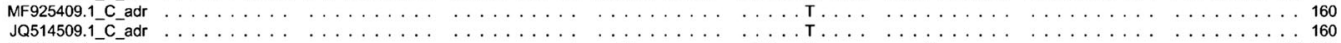

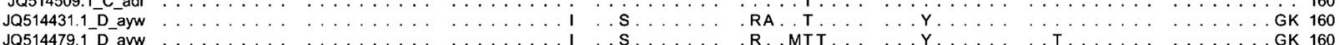

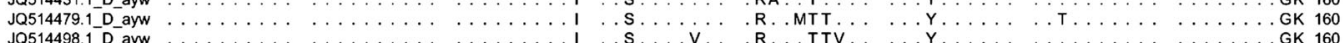

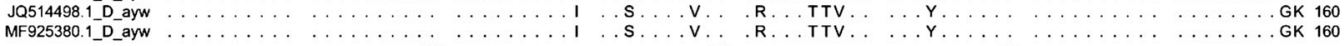
J0514423.1_C_adr FLWEWASVRF SWFSLLVPFV OWFVGLSPTV WLSVIWMMWYY WGPSLHNILN PFLPLLPIFF CLWVYI 220

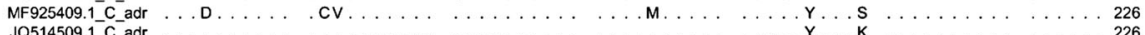

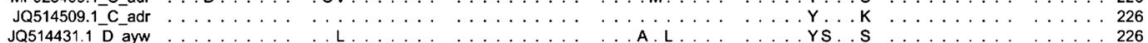

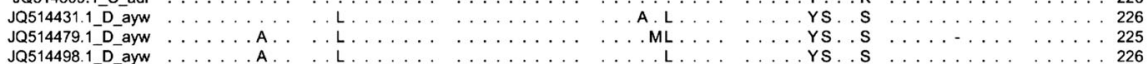

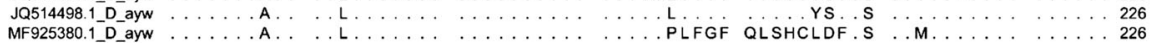

C

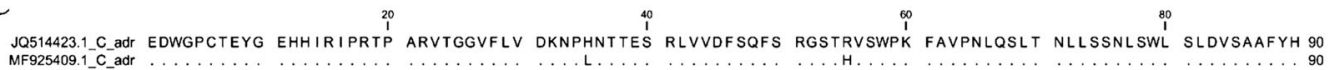

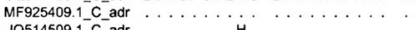

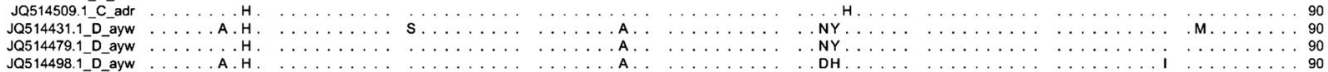

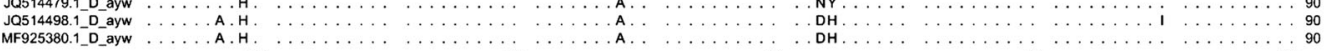
100
Ja514423.1_C_adr LPLHPAMPH LLVGSGLPR YVARLSSTSR NINYAHGTMQ DLHESCSRNL
MF925409.1C_adr

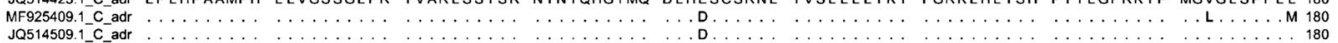

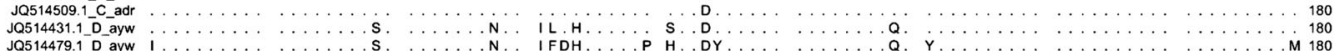

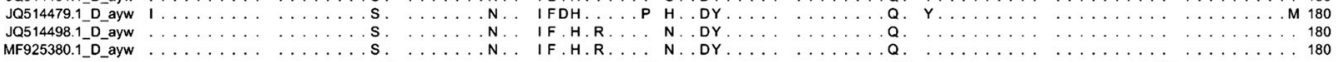
220

240

Ja514423.1 C.Cad VOFTSAICSV VRRAFPHCLA FSYMDOVVLG AKSAQHLESL FTSITNFLLS LGIHLNPNKT KRWGYSLNFM GYVIGSWGTL PQEHIVQKLK 270

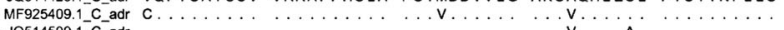
Jo514509.1 C_ _adr Jo514431.1 D Dayw A

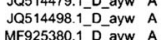

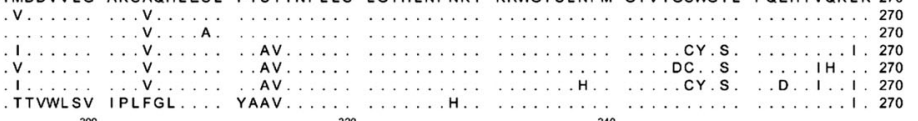

Ja514423.1_C_adr OCFRKLPINR PIOWKVCQRI VGLLGFAAPF TQCGYPALMP LYACIQAKOA 190

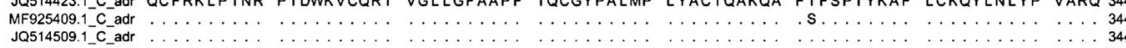
JQ5 14431.1 D_ayw E

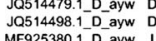
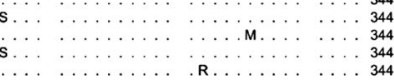

Fig. 5 Alignment of the nucleotide sequences (a) and amino acid sequences of HBsAg, (b) and Pol RT domain (c) of the sequences showed both $\mathrm{HBsAg}$ escape and drug-resistant mutations 
HBsAg escape mutations were found in genotype D followed by genotype $C$ and very less in genotype $A$. The predominant mutation is $128 \mathrm{~V}$ found only in genotype D (Serotype ayw3) though amino acid position 128 is not considered for serotype determinations [25, 42]. HBsAg $128 \mathrm{~V}$ mutation is associated with occult HBV infection found in genotype D [43]. These results would be very useful for the analysis of HBV genome sequences in the future including epitope designing for the diagnostic kit and vaccine development. On the other hand, drug-resistant mutations were highest in genotype $\mathrm{C}$ followed by genotype D with no report in genotype A in this analysis. However, there were no significant differences or correlation among the HBsAg escape and drugresistant mutations.

In summary, there is a strong correlation between HBV genotype and serotype with the prevalence of genotypes C, D, and A is $46.39,35.05$, and $18.56 \%$, respectively, in Bangladesh. The genotypes A, C, and D correspond to subtype $a d w, a d r$, and $a y w$, respectively. HBsAg escape mutations were found around 51\% in Bangladeshi HBV isolates among which $62 \%$ in genotype D followed by $32 \%$ in $\mathrm{C}$ and only $6 \%$ in $\mathrm{A}$ whereas drug-resistant mutation is common in genotypes $\mathrm{C}$ and D. Sequences showing Lamivudine resistant mutations are higher and Tenofovir is susceptible for all sequences in Bangladesh.

\section{Conclusion}

A strong correlation found in this meta-analysis among the HBV genotype/serotype and HBsAg escape and/or drug-resistant mutations that would be very helpful for genotype-serotype prediction by PCR-based diagnosis and development of vaccine and/or diagnostic kits, and the treatment against HBV infection in future. Drugresistant mutational analysis supports the use of tenofovir for HBV treatment in Bangladesh.

\section{Additional file}

Additional file 1. List of accession numbers of the sequences downloaded from NCBI GenBank and used for analysis in this study. The $\mathrm{HBsAg}$ escape and drug-resistant mutational analysis were performed using Geno2pheno-HBV and HIV-Grade:HBV-Tool.

\begin{abstract}
Abbreviations
DNA: Deoxyribonucleic acid; ELISA: Enzyme-linked immunosorbent assay; HBIG: Hepatitis B immunoglobulin; HBsAg: Hepatitis B surface protein; HBV: Hepatitis B virus; HCC: Hepatocellular carcinoma; MHR: Major hydrophilic region; NCBI: National Center for Biotechnology Information; PCR: Polymerase chain reaction
\end{abstract}

\section{Acknowledgments}

Not applicable.

\section{Authors' contributions}

MGH extracted the data from NCBI GenBank, analyzed the data, and wrote the manuscript. KU and MGH edited and revised the manuscript. Both authors read and approved the final manuscript.

\section{Funding}

This study has not been supported by any research grant.

\section{Availability of data and materials}

All the data analyzed in this meta-analysis has been downloaded from the free online database NCBI GenBank which are included in this published article and its supplementary information file.

Ethics approval and consent to participate

The data analyzed in this review were downloaded from the free online database NCBI GenBank

\section{Consent for publication}

No personal data were used in this review.

\section{Competing interests}

The authors declare that they have no competing interests.

\section{Author details}

'Division of Virology, Department of Microbiology and Immunology, Graduate School of Medicine, Osaka University, Osaka, Japan. ${ }^{2}$ Department of Microbiology and Hygiene, Bangladesh Agricultural University, Mymensingh, Bangladesh.

Received: 18 October 2019 Accepted: 29 October 2019

Published online: 07 November 2019

\section{References}

1. Stevens CE, Beasley RP, Tsui J, Lee WC. Vertical transmission of hepatitis B antigen in Taiwan. N Engl J Med. 1975;292(15):771-4

2. Gray Davis L, Weber D, Lemon S. HORIZONTAL TRANSMISSION OF HEPATITIS B VIRUS. Lancet. 1989:333(8643):889-93.

3. Romeo R, Petruzziello A, Pecheur El, Facchetti F, Perbellini R, Galmozzi E, Khan NU, Di Capua L, Sabatino R, Botti G, et al. Hepatitis delta virus and hepatocellular carcinoma: an update. Epidemiol Infect. 2018;146(13):1612-8.

4. Carman WF. The clinical significance of surface antigen variants of hepatitis B virus. J Viral Hepat. 1997:4(Suppl 1):11-20.

5. Magnius LO, Norder H. Subtypes, genotypes and molecular epidemiology of the hepatitis B virus as reflected by sequence variability of the S-gene. Intervirology. 1995;38(1-2):24-34.

6. Yamanaka T, Akahane Y, Suzuki H, Okamoto H, Tsuda F, Miyakawa Y, Mayumi M. Hepatitis B surface antigen particles with all four subtypic determinants: point mutations of hepatitis B virus DNA inducing phenotypic changes or double infection with viruses of different subtypes. Mol Immunol. 1990:27(5):443-9.

7. Gerlich WH. Prophylactic vaccination against hepatitis B: achievements, challenges and perspectives. Med Microbiol Immunol. 2015;204(1):39-55.

8. Hayden CA, Fischer ME, Andrews BL, Chilton HC, Turner DD, Walker JH, Tizard IR, Howard JA. Oral delivery of wafers made from HBsAg-expressing maize germ induces long-term immunological systemic and mucosal responses. Vaccine. 2015;33(25):2881-6.

9. Harrison A, Lemey P, Hurles M, Moyes C, Horn S, Pryor J, Malani J, Supuri M, Masta A, Teriboriki B, et al. Genomic analysis of hepatitis $B$ virus reveals antigen state and genotype as sources of evolutionary rate variation. Viruses. 2011;3(2):83-101

10. Tian Y, Xu Y, Zhang Z, Meng Z, Qin L, Lu M, Yang D. The amino acid residues at positions 120 to 123 are crucial for the antigenicity of hepatitis $B$ surface antigen. J Clin Microbiol. 2007:45(9):2971-8.

11. Sheldon J, Rodes B, Zoulim F, Bartholomeusz A, Soriano V. Mutations affecting the replication capacity of the hepatitis B virus. J Viral Hepat. 2006; 13(7):427-34.

12. Huang $\mathrm{CH}$, Yuan Q, Chen PJ, Zhang YL, Chen CR, Zheng QB, Yeh SH, Yu H, Xue $Y$, Chen $Y X$, et al. Influence of mutations in hepatitis $B$ virus surface protein on viral antigenicity and phenotype in occult HBV strains from blood donors. J Hepatol. 2012:57(4):720-9.

13. Shi Y, Wei F, Hu D, Li Q, Smith D, Li N, Chen D. Mutations in the major hydrophilic region (MHR) of hepatitis B virus genotype C in North China. J Med Virol. 2012;84(12):1901-6.

14. Yu DM, Li XH, Mom V, Lu ZH, Liao XW, Han Y, Pichoud C, Gong QM, Zhang $\mathrm{DH}$, Zhang $\mathrm{Y}$, et al. N-glycosylation mutations within hepatitis $\mathrm{B}$ virus 
surface major hydrophilic region contribute mostly to immune escape. J Hepatol. 2014;60(3):515-22.

15. Cento V, Mirabelli C, Dimonte S, Salpini R, Han Y, Trimoulet P, Bertoli A, Micheli V, Gubertini G, Cappiello G, et al. Overlapping structure of hepatitis $B$ virus (HBV) genome and immune selection pressure are critical forces modulating HBV evolution. J Gen Virol. 2013;94(Pt 1):143-9.

16. Yano Y, Azuma T, Hayashi Y. Variations and mutations in the hepatitis B virus genome and their associations with clinical characteristics. World J Hepatol. 2015;7(3):583-92.

17. Menéndez-Arias L, Álvarez M, Pacheco B. Nucleoside/nucleotide analog inhibitors of hepatitis B virus polymerase: mechanism of action and resistance. Curr Opin Virol. 2014;8:1-9.

18. Monto A, Schooley RT, Lai JC, Sulkowski MS, Chung RT, Pawlotsky JM, McHutchison JG, Jacobson IM. Lessons from HIV therapy applied to viral hepatitis therapy: summary of a workshop. Am J Gastroenterol. 2010;105(5): 989-1004 quiz 1988, 1005.

19. Su M, Xiang K, Li Y, Li Y, Deng J, Xu X, Yan L, Zhuang H, Li T. Higher detection rates of amino acid substitutions in HBV reverse transcriptase/ surface protein overlapping sequence is correlated with lower serum HBV DNA and HBsAg levels in HBeAg-positive chronic hepatitis B patients with subgenotype B2. Infect Genet Evol. 2016;40:275-81.

20. Mahtab MA, Rahman S, Karim MF, Khan M, Foster G, Solaiman S, Afroz S Epidemiology of hepatitis B virus in Bangladeshi general population. Hepatobiliary Pancreat Dis Int. 2008;7(6):595-600.

21. Uz-Zaman MH, Rahman A, Yasmin M. Epidemiology of Hepatitis B Virus Infection in Bangladesh: Prevalence among General Population, Risk Groups and Genotype Distribution. Genes. 2018;9(11):541.

22. Munshi SU, Tran TTT, Vo TNT, Tabassum S, Sultana N, Nguyen TH, Jahan M, Le CN, Baker S, Rahman M. Molecular characterization of hepatitis B virus in Bangladesh reveals a highly recombinant population. PLoS One. 2017; 12(12):e0188944.

23. Rahman MA, Hakim F, Ahmed M, Ahsan CR, Nessa J, Yasmin M. Prevalence of genotypes and subtypes of hepatitis B viruses in Bangladeshi population. SpringerPlus. 2016;5:278.

24. Shaha M, Hoque SA, Rahman SR. Molecular epidemiology of hepatitis $B$ virus isolated from Bangladesh. SpringerPlus. 2016;5(1):1513.

25. Bell TG, Kramvis A. Bioinformatics tools for small genomes, such as hepatitis $B$ virus. Viruses. 2015:7(2):781-97.

26. Beggel B, Neumann-Fraune M, Doring M, Lawyer G, Kaiser R, Verheyen J, Lengauer T. Genotyping hepatitis B virus dual infections using populationbased sequence data. J Gen Virol. 2012;93(Pt 9):1899-907.

27. Neumann-Fraune M, Beggel B, Kaiser R, Obermeier M. Hepatitis B virus drug resistance tools: one sequence, two predictions. Intervirology. 2014;57(3-4): 232-6.

28. Tangkijvanich $\mathrm{P}$, Mahachai $\mathrm{V}$, Komolmit $\mathrm{P}$, Fongsarun J, Theamboonlers A, Poovorawan Y. Hepatitis B virus genotypes and hepatocellular carcinoma in Thailand. World J Gastroenterol. 2005;11(15):2238-43.

29. Noor-E-Alam SM. Management of hepatocellular carcinoma: Bangladesh perspective. Eur J Hepato-Gastroenterol. 2018;8(1):52-3.

30. Kramvis A, Arakawa K, Yu MC, Nogueira R, Stram DO, Kew MC. Relationship of serological subtype, basic core promoter and precore mutations to genotypes/subgenotypes of hepatitis B virus. J Med Virol. 2008;80(1):27-46.

31. Moriyama M, Taira M, Matsumura H, Aoki H, Mikuni M, Kaneko M, Shioda A, Iwaguchi K, Arai S, Ichijima S, et al. Genotype analysis, using PCR with typespecific primers, of hepatitis $B$ virus isolates from patients coinfected with hepatitis delta virus genotype II from Miyako Island, Japan. Intervirology. 2003:46(2):114-20

32. Lin CL, Kao JH. Hepatitis B virus genotypes and variants. Medicine. 2015;5(5): a021436.

33. Le Bouvier GL, McCollum RW, Hierholzer WJ Jr, Irwin GR, Krugman S, Giles JP. Subtypes of Australia antigen and hepatitis-B virus. Jama. 1972;222(8):928-30.

34. Tiollais P, Pourcel C, Dejean A. The hepatitis B virus. Nature. 1985;317(6037): 489-95.

35. Alavian SM, Carman WF, Jazayeri SM. HBsAg variants: diagnostic-escape and diagnostic dilemma. J Clin Virol. 2013;57(3):201-8.

36. Yamamoto K, Horikita M, Tsuda F, Itoh K, Akahane Y, Yotsumoto S, Okamoto $H$, Miyakawa Y, Mayumi M. Naturally occurring escape mutants of hepatitis $B$ virus with various mutations in the $S$ gene in carriers seropositive for antibody to hepatitis B surface antigen. J Virol. 1994;68(4):2671-6.
37. Hossain MG, Ueda K. Investigation of a novel hepatitis B virus surface antigen (HBsAg) escape mutant affecting immunogenicity. PLoS One. 2017; 12(1):e0167871.

38. Carman WF, Zanetti AR, Karayiannis P, Waters J, Manzillo G, Tanzi E, Zuckerman AJ, Thomas HC. Vaccine-induced escape mutant of hepatitis B virus. Lancet (London, England). 1990;336(8711):325-9.

39. Cooreman MP, Leroux-Roels G, Paulij WP. Vaccine- and hepatitis B immune globulin-induced escape mutations of hepatitis B virus surface antigen. J Biomed Sci. 2001;8(3):237-47.

40. Foy MC, Thio CL, Hwang HS, Saulynas M, Hamilton JP, Fine DM, Atta MG. False-negative hepatitis B virus (HBV) surface antigen in a vaccinated dialysis patient with a high level of HBV DNA in the United States. Clin Vaccine Immunol. 2012;19(5):820-2.

41. Mahtab MA, Chaudhury M, Uddin MH, Noor-E Alam SM, Rahim MA, Alam MA, Moben AL, Khondaker FA, Choudhury MF, Sarkar MJ, et al. Cost assessment of hepatitis B virus-related hepatitis in Bangladesh. Eur J Hepato-Gastroenterol. 2016;6(2):163-6.

42. Purdy MA, Talekar G, Swenson P, Araujo A, Fields H. A new algorithm for deduction of hepatitis $B$ surface antigen subtype determinants from the amino acid sequence. Intervirology. 2007;50(1):45-51.

43. Kumar GT, Kazim SN, Kumar M, Hissar S, Chauhan R, Basir SF, Sarin SK. Hepatitis B virus genotypes and hepatitis B surface antigen mutations in family contacts of hepatitis B virus infected patients with occult hepatitis B virus infection. J Gastroenterol Hepatol. 2009;24(4):588-98.

\section{Publisher's Note}

Springer Nature remains neutral with regard to jurisdictional claims in published maps and institutional affiliations.

Ready to submit your research? Choose BMC and benefit from:

- fast, convenient online submission

- thorough peer review by experienced researchers in your field

- rapid publication on acceptance

- support for research data, including large and complex data types

- gold Open Access which fosters wider collaboration and increased citations

- maximum visibility for your research: over $100 \mathrm{M}$ website views per year

At $\mathrm{BMC}$, research is always in progress.

Learn more biomedcentral.com/submissions 